\title{
Pro Kortikoidschutz bei schwerer Pneumonie
}

Müssen Patienten wegen schwere Pneumonie stationär behandelt werden, senkt die Zusatztherapie mit Kortikoiden das Sterberisiko und die Gefahr einer respiratorischen Insuffizienz, auch können die Patienten früher nach Hause. So das Ergebnis einer Metaanalyse.

Bei Pneumonie hilft eine systemische Entzündung, den bakteriellen Angriff zu beherrschen. Allerdings beeinträchtigt sie auch den alveolären Gasaustausch, kann so ein akutes Atemnotsyndrom (ARDS) begünstigen und zur Sepsis führen. Ob eine systemische Kortikoidtherapie (SKT) dies bei hospitalisierten Patienten mit ambulant erworbener Pneumonie (community-acquired pneumonia, CAP) verhindert, ist aber umstritten. In den meisten Leitlinien wird die Zusatztherapie mit Kortikoiden nicht erwähnt. Nun liegt aber eine Reihe kontrollierter Interventionsstudien zur CAP-Therapie vor, z.T. mit widersprüchlichen Ergebnissen. Solche Studien haben Epidemiologen um Dr. Reed Siemieniuk von der Universität Hamilton nun ausgewertet. Fazit: Unterm Strich deuten die Studien auf einen deutlichen Nutzen der SKT hin.

Die Forscher fanden 13 kontrollierte Studien mit oraler oder i.v. Kortikoidtherapie bei CAP. An diesen hatten sich über 2000 Patienten beteiligt. Patienten mit hohem Risiko für KortikoidNebenwirkungen waren meist ausgeschlossen. In zwölf der Studien war die Sterberate ermittelt worden. Mit Kortikoiden zusätzlich zur Antibiose starben 5,3\% der Patienten, mit Antibiotika plus Placebo waren es 7,9\%. Die Sterberate konnte somit absolut um 2,6 \%-Punkte und relativ um ein Drittel verringert werden. Der Vorteil bei der Mortalität ließ sich aber nur bei schwerer Pneumonie nachweisen, hier berechnete man eine Risikoreduktion um $61 \%$. Meist galten Patienten als schwer krank, die eine mechanische Beatmung, Vasopressoren oder beides benötigten. Aufgrund des Subgruppeneffekts und der recht großen Streuung der Ergebnisse der einzelnen Studien beurteilen die Forscher um Siemieniuk den Effekt auf die Mortalität als moderat und sprechen von einer „möglichen Reduktion“ der Gesamtsterberate.

Etwas ausgeprägter scheint der Effekt der SKT zu sein, wenn es darum geht, eine mechanische Beatmung zu vermeiden. Relativ betrachtet wurde diese unter Kortikoiden zu 55\% seltener benötigt, absolut lag der Unterschied bei 5\%-Punkten. Da aber nur in 5 Studien danach geschaut wurde und nur 46 Patienten überhaupt mechanisch beatmet wurden, nennen die Forscher auch hier die Evidenz für einen Nutzen der SKT moderat.

Patienten mit Kortikoiden mussten seltener auf die Intensivstation, die Rate war um ca. ein Drittel geringer als mit Placebo. Nur 3 Studien schauten danach, die Forscher um Siemieniuk sehen hier ebenfalls eine moderate Evidenz zugunsten der SKT.

In vier Studien fanden die Forscher Angaben zur ARDS-Rate. Diese war mit SKT um 76\% geringer. Allerdings trat ein ARDS nur bei 16 Patienten auf, was die Aussagekraft schmälert.

In den Untersuchungen lassen sich auch Hinweise finden, wonach SKT die Behandlungsdauer verkürzt. So wurden in Studien mit geringer Fehlerwahrscheinlichkeit die Patienten mit SKT einen Tag früher stabilisiert (klinische Zeichen normal, kein zusätzlicher Sauerstoff) und einen Tag früher entlassen. Die Evidenz dafür wertete die Forscher als hoch. Allerdings begünstigt die SKT sehr wahrscheinlich eine therapierelevante Hyperglykämie, die Rate dafür war mit SKT relativ um 50\%, absolut um 4\% erhöht.

Noch unklar ist, welche Kortikoide in welcher Dosis und für wie lange den besten Nutzen für CAP-Patienten haben. Hierfür sind wohl weitere kontrollierte Studien nötig. Eine solche Studie mit der Bezeichnung ESCAPe (Extended Steroid in CAP) läuft derzeit mit Methylprednisolon (20 und $40 \mathrm{mg} / \mathrm{d}$ ). Thomas Müller

Quelle: springermedizin.de basierend auf: Siemieniuk RAC et al. Ann Intern Med, online 11.8.2015; doi:10.7326/M15-0715

\section{Künstliche Beatmung zu Hause: Modellprojekt ausgezeichnet}

Wenn ein künstlich beatmeter Patient aus der Klinik nach Hause geht, ist das für Ärzte und Pfleger eine Herausforderung. Nun werden in einem Modellprojekt neue Versorgungskonzepte erprobt, das einen Preis erhielt.

Sie sind noch recht neu in der ambulanten Versorgung, aber es werden immer mehr: Sie leiden an einer Chronisch Obstruktiven Lungenerkrankung (COPD), an neuromuskulären Erkrankungen, einer Querschnittslähmung, einem Schlaganfall, Parkinson oder Multipler Sklerose. Sie sind auf künstliche Beatmung angewiesen und leben trotzdem in ihrer häuslichen Umgebung. "Bis vor wenigen Jahren waren diese Patienten nur im Krankenhaus. Mittlerweile gehen sie direkt von der Intensivstation in die Häuslichkeit", sagt der Pneumologe Dr. Eckehard Frisch vom Berliner Centrum für Gesundheit (CfG), ein Ärztezentrum der AOK Nordost.

Doch die ambulante Versorgung stößt bei diesen Patienten oft an ihre Grenzen. „Ärztliche Versorgung von außerklinisch beatmeten Patienten findet im Wesentlichen durch Hausärzte, punktuell durch Fachärzte statt, welche kaum Erfahrung im Bereich der außerklinischen Beatmung haben. Damit sind die Patienten gemessen an der Schwere und Komplexität ihrer Erkrankung unterversorgt", so Frisch. Das führt laut AOK Nordost dazu, dass
Betroffene wieder stationär eingeliefert werden müssen. Nach Frischs Angaben gibt es auch viele, die länger beatmet werden als nötig. Die Krankenkasse hat daher mit Frisch einen Modellversuch mit dem Titel "Praxis für außerklinische Beatmung" gestartet. Dieses Projekt hat die Deutsche Interdisziplinäre Gesellschaft für Außerklinische Beatmung (DIGAB) nun mit dem 1. Preis für das „Beste Abstract” ausgezeichnet. Das auf 2 Jahre angelegte Projekt soll die Therapie der beatmeten Patienten durch Hausbesuche von Spezialisten verbessern. Mindestens einmal pro Quartal kommen ein Pneumologe und eine Atmungstherapeutin zu den Patienten. Zudem sind sie Werktagen für Patienten, Angehörige und andere Beteiligte erreichbar. Auch wenn nicht genug Windeln da sind oder der Rolli kaputt ist, kümmert sich das Team. Dabei legt Frisch Wert auf die Zusammenarbeit mit Hausarzt, Logopäden, Physiotherapeuten, Pflege und Hilfsmittellieferanten. „Wir sind kein Ersatz für den Hausarzt, sondern eine Ergänzung. Wir sehen uns als Unterstützung und versuchen die Versorgung als Teamspiel aufzustellen", so Frisch. Oft geht es auch um die Versorgung am Lebensende. Da ist es von Vorteil, dass Frisch auch Palliativmediziner ist. Quelle: SAPV; Ärzte Zeitung, 3.9.2015 